\title{
Verification of a Motion Cueing Strategy for Stall Recovery Training in a Commercial Transport Simulator
}

\author{
AIAA-2019-0426
}

Peter M. T. Zaal ${ }^{1}$ William W. Chung ${ }^{2}$ Diane M. Carpenter ${ }^{3}$

Kevin Cunningham ${ }^{4}$ Gautam H. Shah ${ }^{4}$

January 7, 2019

${ }^{1}$ San José State University

NASA Ames Research Center

${ }^{4}$ NASA Langley Research Center
${ }^{2}$ American Systems

NASA Ames Research Center
${ }^{3}$ Flight Research Associates NASA Ames Research Center 


\section{Table of Contents}

1. Introduction

2. Simulator Implementation

3. Experiment Setup

4. Results

5. Conclusions

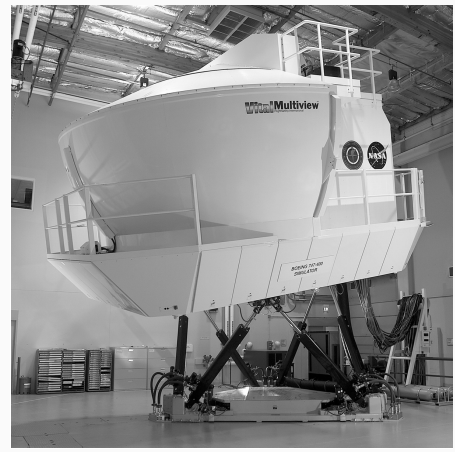




\section{Introduction}

1. Pilots are required to perform full stall recovery training in simulators starting this year

2. Historically, training simulators were not equipped for this

3. Post-stall aircraft models and representative motion cues need to be implemented

\section{Research Goal}

Develop motion cueing strategies for stall recovery training in commercial training simulators 


\section{Motion Cueing Strategy}

1. Simulators have limited motion space

2. Accelerations at pilot station need to be attenuated

3. Center of gravity linear accelerations require most motion space

\section{Approach}

Eliminating the center-of-gravity linear accelerations allows for a significant increase of the fidelity of remaining motion cues

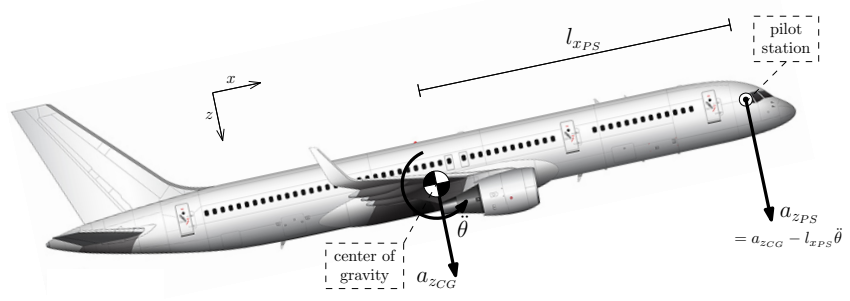




\section{Motion Cueing Strategy}

Limitations:

1. No sustained g-loads

2. No deceleration cue

3. No turn coordination 


\section{B747-400 Full Flight Simulator}

1. Equivalent to level-D certified

2. B747-400 cockpit replica

3. Collimated out-the-window visuals

4. Digital control loading system

5. 54-inch legged hexapod

6. Tabled computer for questionnaire
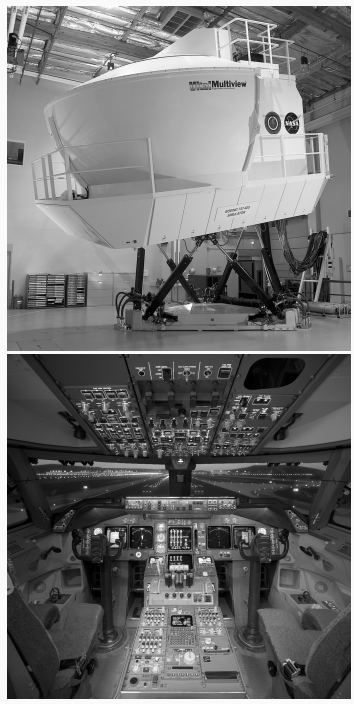


\section{Stall Recovery Task}

1. Initially: $36,000 \mathrm{ft}, 210$ IAS, in the clouds, turbulence

2. Retard throttles to idle and pull up, keeping wings level

3. Continue deceleration through stick shaker until a tone sounds indicating the stall

4. Recover using correct recovery procedure

5. Task evaluation ends when the airspeed is above 210 IAS, the aircraft is climbing, and the wings are level

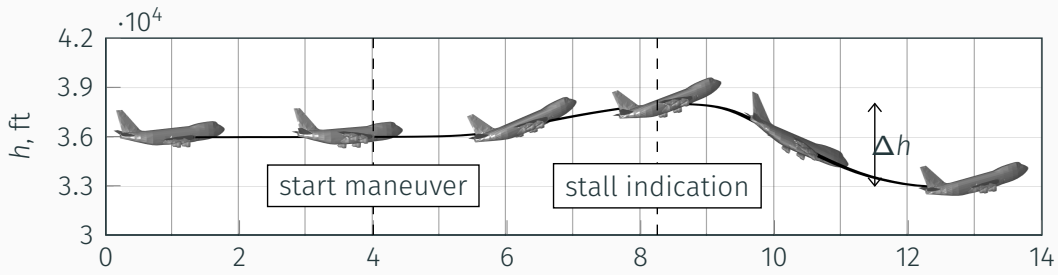




\section{Aircraft Dynamics}

Modification of a very large, generic, four engine transport aircraft:

1. Roll damping stability coefficient

2. Rolling moment increment due to stall asymmetry

3. Aileron effectiveness gain
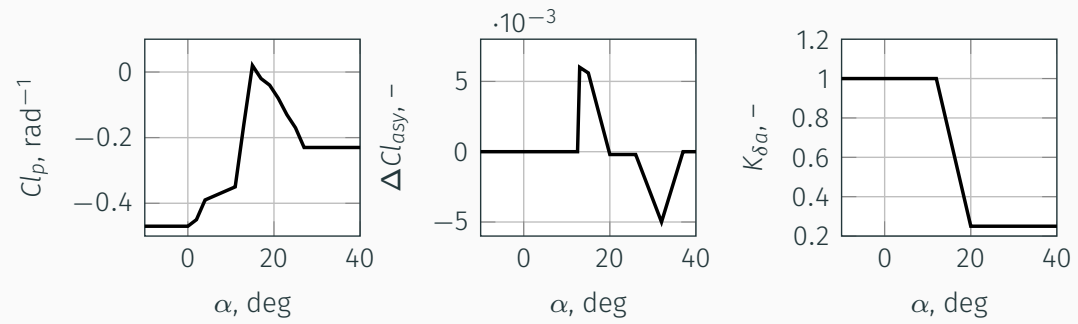


\section{Simulator Motion}

1. Disabling adaptiveness

2. Gains in equation for pilot station accelerations:

$$
a_{p s}=K_{t} a_{c g}+K_{r}\left(\dot{\omega} \times r_{c g-p s}+\omega^{2} \times r_{c g-p s}\right)
$$

3. Online adjustment of motion parameters

\begin{tabular}{lrrrrrr}
\hline \hline & \multicolumn{7}{c}{ Degree of Freedom } \\
\cline { 2 - 7 } & Surge & Sway & Heave & Roll & Pitch & Yaw \\
\hline High-Pass Gains & 0.7 & 0.7 & 0.7 & 1.0 & 1.0 & 1.0 \\
High-Pass Break Frequencies & 0.6 & 0.6 & 0.6 & 0.3 & 0.3 & 0.3 \\
Low-Pass Gains & 1.0 & 1.0 & - & - & - & - \\
Low-Pass Break Frequencies & 0.6 & 0.6 & - & - & - & - \\
\hline \hline
\end{tabular}

Damping ratios $(\zeta)$ :

0.707

C.G. acceleration gain $\left(K_{t}\right): \quad 0.000$

Rot. acceleration gain $\left(K_{r}\right): \quad 1.000$ 


\section{Experiment Setup}

1. Six conditions

2. Latin square design

3. Seven replications per condition (42 runs)

4. Last five replications used for results

\begin{tabular}{ccc}
\hline \hline Condition & Aircraft Dynamics & Simulator Motion \\
\hline B1 & baseline & no motion \\
B2 & baseline & baseline \\
B3 & baseline & enhanced \\
E1 & enhanced & no motion \\
E2 & enhanced & baseline \\
E3 & enhanced & enhanced \\
\hline \hline
\end{tabular}




\section{Experiment Setup}

1. Eight commercial airline pilots

2. Four different airlines

3. Left or right seat

4. No specifics about conditions

5. Post-run questionnaire
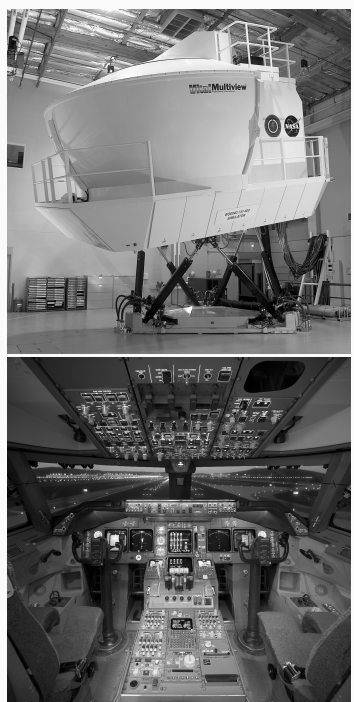


\section{Experiment Setup}

Dependent measures:

1. Four subjective questionnaire responses:

1.1 Motion rating

1.2 Motion usefulness question

1.3 Wing roll-off noticeability question

1.4 Stall recovery in actual flight question

2. Six objective performance measures:

2.1 Maximum roll attitude

2.2 Altitude loss

2.3 Minimum load factor

2.4 Maximum load factor

2.5 Number of secondary stick shakers

2.6 Maximum airspeed 


\section{Results}

Motion rating:

1. No significant differences

2. Enhanced motion rated lower?

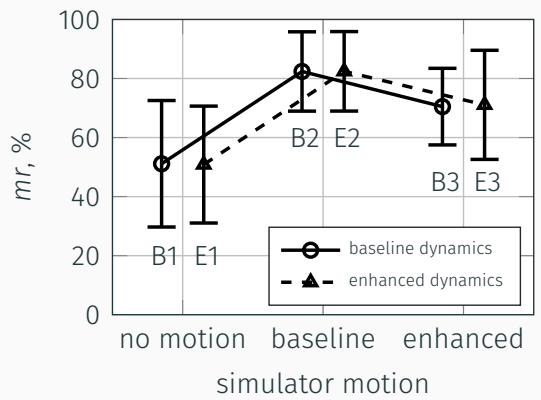

Roll off question:

1. Significantly higher with enhanced dynamics

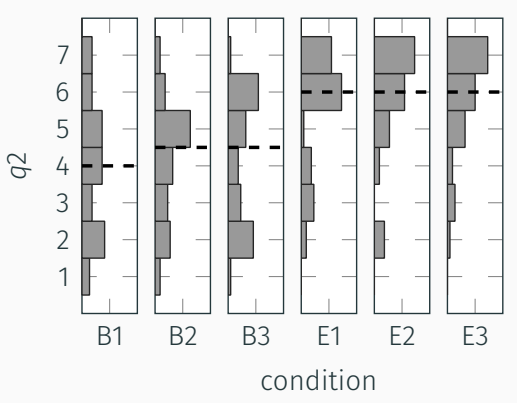




\section{Results}

Maximum roll:

1. Significantly higher with enhanced dynamics

2. Significantly lower with higher fidelity motion

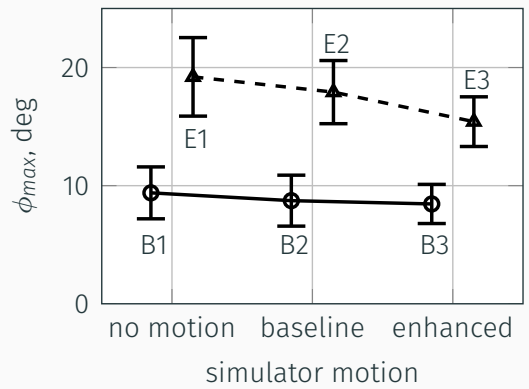

Altitude loss:

1. No significant differences

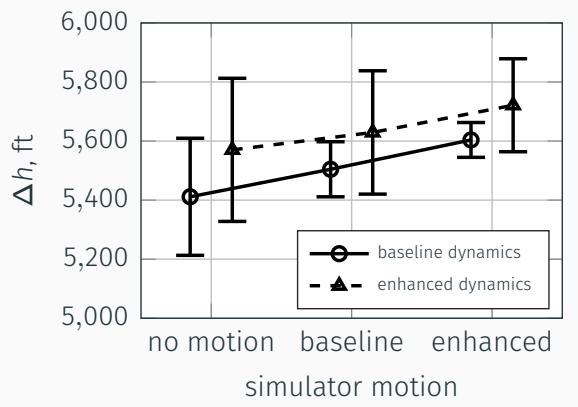




\section{Results}

Minimum load factor:

1. Significantly higher with enhanced motion

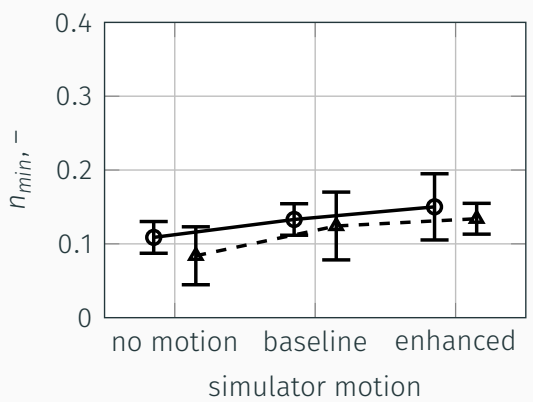

Maximum load factor:

1. No significant differences

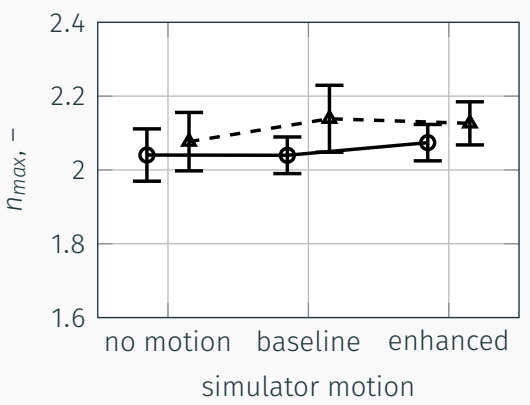




\section{Results}

Additional stick shakers:

1. Significantly lower with enhanced motion

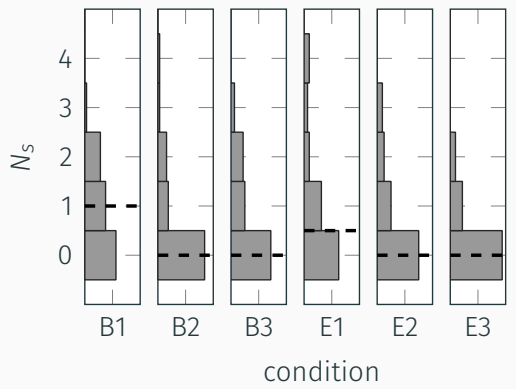

Maximum airspeed:

1. Significantly higher with enhanced motion

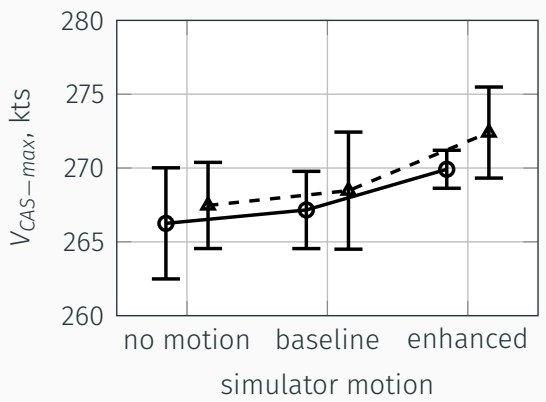




\section{Conclusions}

1. Aircraft dynamics and motion introduced significant differences

1.1 Motion helpfulness question

1.2 Maximum roll

1.3 Additional stick shakers

1.4 Minimum load factor

1.5 Maximum airspeed

2. Better stall recovery performance with enhanced motion

3. Relatively minor enhancements to potentially improve training 
Questions?

peter.m.t.zaal@nasa.gov 